\title{
Rapid evaporation at the superheat limit
}

\author{
By J. E. SHEPHERD AND B. STURTEVANT \\ Graduate Aeronautical Laboratories, California Institute of Technology, \\ Pasadena, CA 91125
}

(Received 6 July 1981 and in revised form 14 December 1981)

In an experimental investigation of the transient processes that occur when a single droplet of butane at the superheat limit vaporizes explosively, short-exposure photographs and fast-response pressure measurements have been used to construct a description of the complete explosion process. It is observed that only a single bubble forms within the drop during each explosion, and that the growth proceeds on a microsecond time scale. An interfacial instability driven by rapid evaporation has been observed on the surface of the bubbles. It is suggested that the Landau mechanism of instability, originally described in connection with the instability of laminar flames, also applies to rapid evaporation at the superheat limit.

The photographic evidence and the pressure data are used to estimate the evaporative mass flux across the liquid-vapour interface after the onset of instability. The rate of evaporation is shown to be two orders of magnitude greater than would be predicted by conventional bubble-growth theories that do not account for the effects of instability. An estimate of the mean density within the bubbles during the evaporative stage indicates that it is more than one half of the critical density of butane.

Additional interesting dynamical effects that are observed include a series of toroidal waves that form on the interface between the butane vapour and the external host liquid in the bubble column apparatus after the bubble has grown large enough to contact the outer edge of the drop, and violent oscillations of the bubble that occur on a millisecond time scale, after evaporation of the liquid butane is complete, that cause the disintegration of the bubble into a cloud of tiny bubbles by Rayleigh-Taylor instability.

\section{Introduction}

In the absence of bubble-forming nuclei, liquids may be heated to temperatures far above their boiling points. There is, however, an absolute limit of superheat, determined by the limit of mechanical stability of fluids, which for a pure substance is $(\partial p / \partial v)_{T}=0$, and by a concomitant exponential growth of the level of phase fluctuations in the substance (Skripov 1974). The limit of superheat is only about $10 \%$ below the critical temperature of many substances, so superheats of more than $100^{\circ} \mathrm{C}$ can in principle be attained. Heating to the superheat limit is made possible by suppressing heterogeneous nucleation and ordinary boiling by, for example, heating or depressurizing the liquid very rapidly (on a microsecond time scale), or by immersing the volatile liquid in another liquid, thus isolating it from rough solid surfaces containing gas nuclei. When such extreme superheats do occur, and boiling begins spontaneously by homogeneous nucleation, the ensuing evaporative fluxes, fluid accelerations 


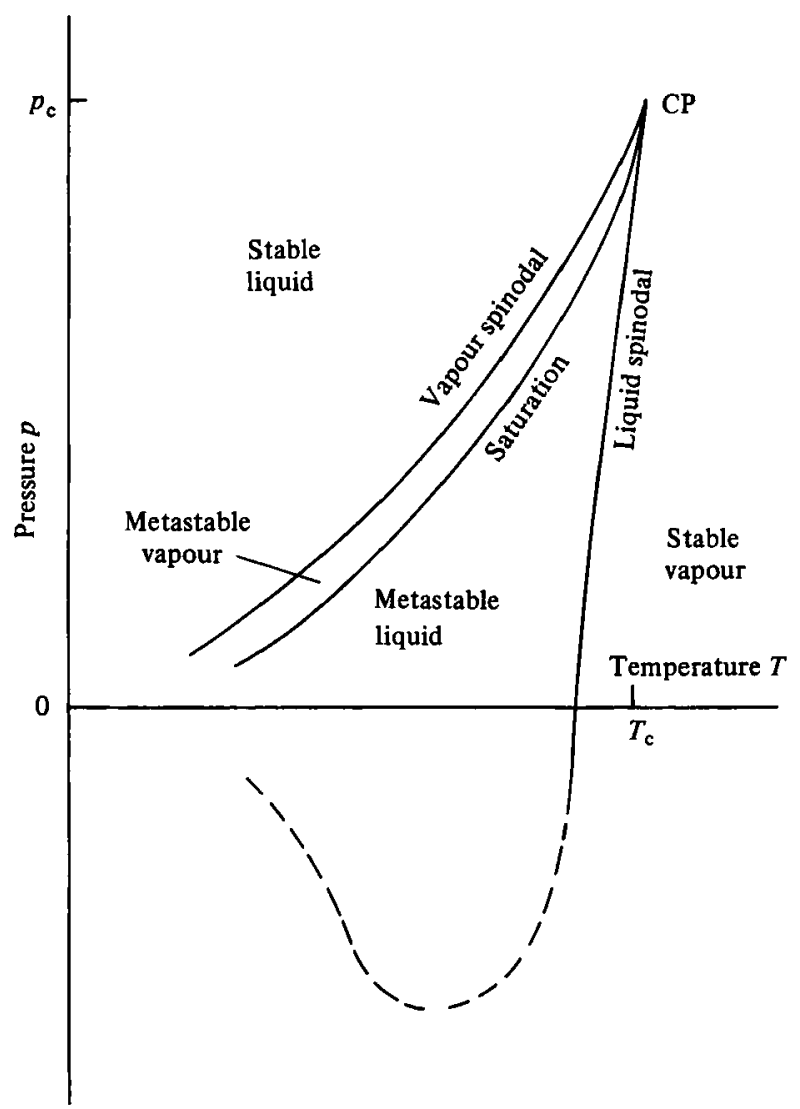

Figure 1. Pressure-temperature diagram showing liquid, vapour and metastable states.

and departures from thermodynamic equilibrium are orders of magnitude greater than in ordinary boiling. The resulting explosive process is known as a vapour explosion, and, when it occurs accidentally in industry or in nature, it can be very destructive.

The locus of points of neutral mechanical stability on a thermodynamic state diagram such as the $p-T$ diagram (figure 1 ) is known as the spinodal. The limit of superheat is observed to occur very near to, if not at, the spinodal (Skripov 1974). A kinetic interpretation of the superheat limit is provided by the theory of homogeneous nucleation, in which the spectrum of bubble sizes in a fluid is calculated on a probabilistic basis using the fluctuation theory of statistical mechanics. The state at which the spectrum of bubbles first contains a large number that are growing is defined as the superheat limit. Indeed, if one properly interprets density fluctuations $\Delta \rho$ as fluctuations of phase, fluctuation theory provides the connection between the limit of stability and this kinetic superheat limit through the result

$$
\frac{\overline{(\Delta \rho)^{2}}}{\rho^{2}}=-\frac{k T}{V} \frac{1}{v}\left(\frac{\partial p}{\partial v}\right)_{T}^{-1},
$$

where $V$ is the volume of the sample, $\rho, v, p$ and $T$ are the density, specific volume, pressure and temperature respectively, and $k$ is Boltzmann's constant.

Vapour explosions as they actually occur in practice involve many complex pro- 
cesses, including fluid mixing, fragmentation, boiling heat transfer, multiphase flow and non-equilibrium evaporation. Accidental vapour explosions are hypothesized to have played a role in explosions in foundries and the paper industry (Witte, Cox \& Bouvier 1970), spillage of liquified natural gas into sea water (Reid 1978), nuclearreactor cooling-system failures (Board \& Caldarola 1977), railway tank car explosions (Reid 1979) and interactions of molten lava with water (Colgate \& Sigurgeirson 1973). Included in the last category are such spectacular examples as the catastrophic explosion of Krakatoa in 1883 and, more recently, of Mt St Helens. The mechanism in all these explosions is thought to be extremely rapid evaporation of liquid at the superheat limit resulting from depressurization or contact with a hotter fluid. Vapour explosions, though often destructive, are generally weaker than chemical explosions because they are endothermic and the stored energy, being thermal, is usually smaller.

A detailed theory accounting for the full complexity of vapour explosions does not exist. It is important that, in attempting to build the understanding required for developing such a theory, the several processes active in vapour explosions listed above be investigated individually and in depth. Most investigations to date have involved medium- or large-scale simulations of accidental spills in which various combinations of molten metals and/or liquids have been injected or poured into one another (e.g. Anderson \& Armstrong 1974). Consequently, it is still impossible to predict from physical reasoning such important parameters as the duration and severity of vapour explosions. This paper reports an experimental investigation of the transient processes that take place just after a single droplet of metastable liquid at the superheat limit begins to boil. The vaporization process and resulting blast-generated pressure field are studied.

One simple configuration in which rapid evaporation can effectively be studied in relative isolation from other complicating factors is the vapour explosion of droplets in the so-called bubble-column apparatus (Wakeshima \& Takata 1958; Moore 1959). In this device the volatile liquid is isolated from possible nucleation centres by immersion in another liquid, a technique first used by $\operatorname{Dufour}(1861 a, b)$, who 120 years ago was able to superheat water by as much as $78^{\circ} \mathrm{C}$ and who remarked at the 'explosion' that could be induced under these circumstances by introducing foreign objects into the drop. Despite the widespread use of this technique for many decades, and despite considerable speculation in the literature about the miniature explosions that are always observed when the droplets vaporize, the fundamental behaviour of the explosion, including such rudimentary details as its time scale and the magnitude of the blast-generated pressure field, has not previously been documented.

In the present experiments, short-exposure photographs and fast-response pressure measurements have been used to obtain a preliminary description of the complete explosion process within superheated drops immersed in the bubble-column apparatus. It is observed that the spontaneous vaporization of superheated drops proceeds by the formation and growth on a microsecond time scale of only one bubble within each drop. Several new and unusual features of the early evaporative stage of the explosion have been observed. For example, photographs of the liquid-vapour interface show a largeamplitude small-scale roughening of the bubble surface during most of the evaporative stage, in stark contrast to the smooth bubbles observed in conventional boiling. The roughening is believed to be the manifestation of a previously undiscovered interfacial instability which is driven by rapid evaporation. A quantitative estimate of the 
evaporative flux across the liquid-vapour interface shows that it is at least two orders of magnitude greater than that predicted by the classical theory of bubble growth, which does not account for the effects of instability. Indeed, this mass flux is of the same order as the maximum possible one-way flux across a smooth interface given by kinetic theory, namely $\frac{1}{4} n \bar{c}$, where $n$ is the number density and $\bar{c}$ is the mean molecular speed, despite the fact that the density of the vapour within the bubble may be as much as $25 \%$ of liquid density, not vacuum! Such large evaporation rates, of course, have grave implications for the severity of vapour explosions.

Some interesting dynamical effects are also observed in these experiments. When the size of the rapidly expanding bubble reaches that of the initial drop, surface waves driven by vapour jetting form on the surface of the bubble, giving it a distinctive appearance. Also, after the liquid has completely evaporated, the bubble oscillates with such violent accelerations (of order $10^{4} \mathrm{~g}$ ) that it rapidly breaks up under RayleighTaylor instability.

The theory referred to as the classical theory of bubble growth in this paper describes the growth of a smooth spherical vapour bubble from a critical nucleus in a uniformly superheated liquid. The most complete version of this theory is due to Prosperetti \& Plesset (1978). It accounts for heat-transfer effects in the liquid, but assumes that the vapour is in thermodynamic equilibrium. That is, it does not treat the evaporative process at the liquid-vapour interface explicitly. Nevertheless, the evaporative mass flux implied by the assumptions of the theory can be calculated (cf. $\$ 3.1 .3$ ).

The classical theory shows that bubble growth proceeds in three stages: first, a surface-tension-controlled stage in which the bubble grows from a critical radius; secondly, an inertia-controlled stage in which the bubble radius grows at constant speed $R$ determined by the vapour pressure and density of the superheated fluid; and thirdly, an asymptotic stage in which bubble growth is limited by heat transfer and follows a $t^{-\frac{1}{2}}$ dependence. Once the bubble has grown out of the surface-tensiondominated stage the growth can be described approximately by a universal equation in scaled variables (Prosperetti \& Plesset 1978). Assumptions made in deriving the universal equation are that (i) the pressure and temperature of the vapour in the bubble are uniform, (ii) the vapour is in equilibrium with the liquid at the temperature $T_{\ell}$ of the liquid surface, (iii) the vapour pressure $p_{\mathrm{v}}\left(T_{\ell}\right)$ is a linear function of the temperature $T_{\ell}$, (iv) the latent heat is constant, and (v) the surface tension is negligible.

In this paper the experimental apparatus and technique are described in $\$ 2$ and the results leading to consideration of evaporative instability are presented in $\S 3$. For completeness, pictures of the formation of surface waves by jetting and of the development of Rayleigh-Taylor instability are also presented. The mechanism for evaporative instability and growth of the bubble after the onset of instability are discussed in $\S 4$.

\section{Experimental}

In the bubble-column apparatus a vertical column of host liquid is maintained hotter at the top than at the bottom, and the substance to be studied is introduced at the bottom in the form of a small liquid drop. The host liquid is selected so the drop liquid is both immiscible and buoyant in it. In addition, the host is chosen so that it 


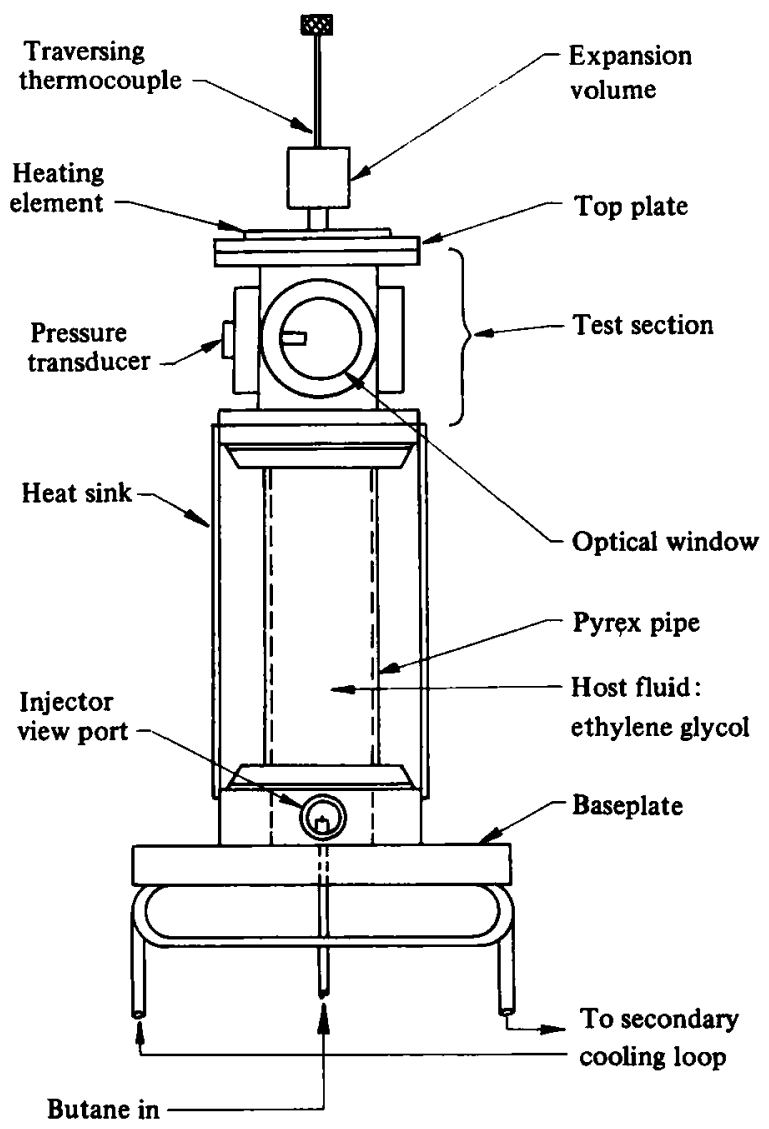

Fiaure 2. Bubble column and test section.

does not itself boil in the temperature range between the boiling point and superheat limit of the drop. The liquid in the droplet is initially below its boiling point, and, as it slowly rises up the column by its buoyancy, it is heated at essentially constant pressure by heat transfer from the surrounding host. For sufficiently small droplets the velocity of rise is slow enough that the temperature distribution within the drop is uniform and close to the local temperature of the host. In this way the drop temperature can be increased to the superheat limit near the top of the column, where homogeneous nucleation occurs and the drop evaporates with an explosive pop. Figure 2 is a schematic diagram of the bubble-column apparatus used in the present experiment. Host and test fluids were chosen from considerations of convenience and the experience of previous investigators (e.g. Porteous \& Blander 1975). Ethylene glycol is used for the host liquid and butane for the test fluid. Typical properties of these substances are given in table 1 .

Butane has a particularly convenient boiling point $\left(-0.5^{\circ} \mathrm{C}\right)$, and its superheat limit $\left(105^{\circ} \mathrm{C}\right)$ is low enough that conventional piezoelectric transducers may be immersed in the test section with no risk of depolarizing the sensitive element. The test section, which is $10 \mathrm{~cm}$ square, was made as large as possible to delay the effects of pressure waves reflecting from its walls and arriving back at the site of the explosion during the measurements. The bubble column is mounted on a refrigerated base plate. 


\begin{tabular}{|c|c|c|c|c|}
\hline Property & Symbol & Butane & Ethylene glycol & Units \\
\hline Critical temperature & $T_{\mathrm{o}}$ & 156 & 372 & ${ }^{\circ} \mathrm{C}$ \\
\hline Superheat limit & $T_{\mathrm{g}}$ & 105 & & ${ }^{\circ} \mathrm{C}$ \\
\hline Boiling temperature & $T_{\mathrm{b}}$ & -0.5 & $197 \cdot 6$ & ${ }^{\circ} \mathrm{C}$ \\
\hline Freezing temperature & $T_{t}^{0}$ & $-138 \cdot 3$ & -13 & ${ }^{\circ} \mathrm{C}$ \\
\hline Density $\left(20^{\circ} \mathrm{C}\right)$ & $\rho$ & 0.579 & $1 \cdot 11$ & $\mathrm{~g} / \mathrm{cm}^{3}$ \\
\hline Specific heat $\left(20^{\circ} \mathrm{C}\right)$ & $c_{p}$ & $\mathbf{2 \cdot 3 9}$ & $2 \cdot 34$ & $\mathrm{~J} / \mathrm{g}^{\circ} \mathrm{C}$ \\
\hline Thermal conductivity $\left(20^{\circ} \mathrm{C}\right)$ & $k$ & $1 \cdot 18 \times 10^{-3}$ & $2.8 \times 10^{-3}$ & $\mathrm{~W} / \mathrm{cm}^{\circ} \mathrm{C}$ \\
\hline $\begin{aligned} & \text { Viscosity }\left(20^{\circ} \mathrm{C}\right) \\
&\left(100^{\circ} \mathrm{C}\right)\end{aligned}$ & $\mu$ & $0 \cdot 17$ & $\begin{array}{r}20 \cdot 9 \\
2 \cdot 0\end{array}$ & $\begin{array}{l}\mathbf{c P} \\
\mathbf{c P}\end{array}$ \\
\hline Index of refraction & $n$ & $1 \cdot 33$ & $1 \cdot 43$ & \\
\hline Sound speed $\dagger$ & $a$ & 410 & 1463 & $\mathrm{~m} / \mathrm{s}$ \\
\hline Acoustic impedance $\dagger$ & $\rho a$ & $0.191 \times 10^{3}$ & $1.54 \times 10^{5}$ & $\mathrm{~g} / \mathrm{cm}^{2} \mathrm{~s}$ \\
\hline
\end{tabular}

TABLE 1. Physical properties of liquid butane and ethylene glycol

Droplets of butane are injected at the centre of the plate through a $0.2 \mathrm{~mm}$ diameter hypodermic needle. The top plate of the test section is heated with an electric heating element. In steady state operation a nearly linear temperature gradient is maintained in the bubble column.

No special precautions are taken to ensure purity of the test liquids used in these experiments because, as has been shown recently by Buivid \& Sussman (1978), the presence of particulate matter in the test fluid does not a prior $i$ have significant effect on homogeneous nucleation; only particles that carry vapour nuclei, an unlikely event in our experiments, are important. Indeed, the superheat limit observed in the present experiments $\left(105^{\circ} \mathrm{C}\right)$ is the same as reported in other published work. Also, in the present experiments the heating of the butane is not ideal, because the viscosity of ethylene glycol decreases by a factor of 30 from the bottom of the column to the top, so a typical $0.5-1.0 \mathrm{~mm}$ diameter drop attains a Reynolds number of $50-100$ by the time it reaches the test section. Thus, though there is considerable convective mixing within the drop, the mean temperature of the drop lags behind that of the surrounding fluid, and the liquid in the drop is not uniformly heated. This has the consequence that in these experiments nucleation is always observed to occur near the boundary of the drop. However, a series of photographs of small bubbles shortly after nucleation, viewed simultaneously from two directions at right angles, does not show any bubbles that with certainty have nucleated on the drop boundary, but does show some that have certainly nucleated within the drop, though never at the centre. Thus, it is concluded that the boiling observed in these experiments initiates by homogeneous nucleation in bulk liquid.

For all quantitative measurements of pressure, a $6 \mathrm{~mm}$ diameter pressure transducer is flush-mounted in a $6 \mathrm{~cm}$ diameter baffle about $2.5 \mathrm{~cm}$ from the location of the explosion at a position which is free of reflected waves from the walls of the test section for $\sim 60 \mu \mathrm{s}$. Diffracted waves from the edge of the baffle terminate the acquisition of quantitative pressure data at times greater than $30 \mu \mathrm{s}$. For later times the test section acts as a lossy resonant cavity and, though the acoustic source is small and 


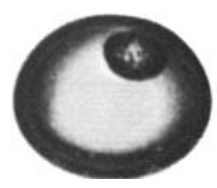

(a)

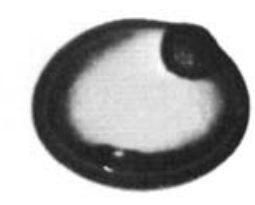

(b)

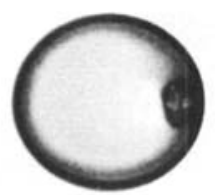

(c)

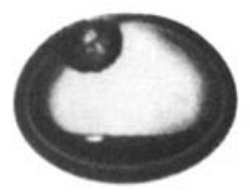

(d)

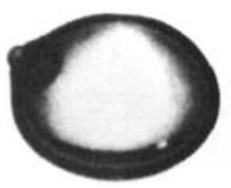

(e)

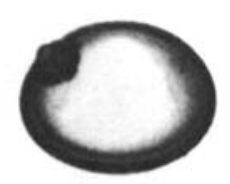

(f)

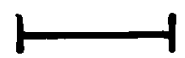

Figure 3. Vapour bubbles in drops at the earliest observed times $(9-12 \mu \mathrm{s})$. Scale indicates $1 \mathrm{~mm}$.

radiates spherical waves which rapidly attenuate radially, the observed wave forms may be affected by reflections from the column walls.

In order to form the optical image of a vapour bubble within a small drop of butane immersed in ethylene glycol, for which the indices of refraction differ greatly (cf. table 1), it is important that the system be illuminated with very diffuse light, that the exposure time be very short, and that the magnification be as large as possible. These criteria are met by using a short-duration ( $<1 \mu \mathrm{s}$ ), high-pressure (1 atm) air/argon spark-gap light source behind an efficient tubular diffuser, together with a camera giving a magnification of $2 \times$ on the film. Only one photograph is taken of each explosion. The triggering signal used to fire the light source is derived from the pressure signal generated by the explosion itself, so an inherent delay equal to the time for the pressure signal to travel from the droplet to the transducer $(1 \mathrm{~cm})$ limits the earliest possible time $(8 \mu \mathrm{s})$ after initiation of the explosion that any photographs can be taken. A series of photographs documenting the entire explosion process has been assembled by using different delays between the occurrence of the pressure signal and triggering of the spark gap. In the labels of the photographs and pressure traces in this paper, all times are given in terms of the time elapsed since the pressure wave first left the drop. 


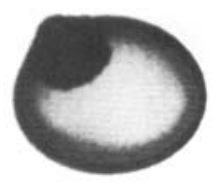

(a)

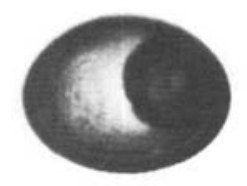

(b)

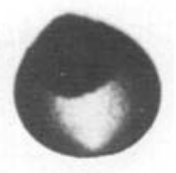

(c)

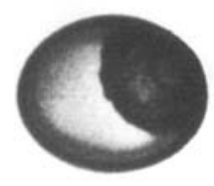

(d)

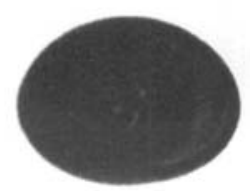

(e)

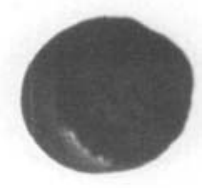

(f)

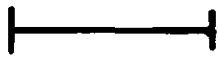

FigURE 4. Growing vapour bubbles, showing developing jet structure and roughened evaporating surfaces $(17-34 \mu \mathrm{s})$. Scale indicates $1 \mathrm{~mm}$.

\section{Results}

\subsection{Rapid evaporation}

3.1.1. Photographs. Figure 3 shows representative examples of photographs of exploding droplets at the earliest time $(\sim 10 \mu \mathrm{s})$ that pictures could be taken after initiation of the vapour explosion by homogeneous nucleation. The drops are shown immersed in host liquid, which appears pure white in the photographs. Within each drop is seen one bubble. The darkening at the edge of the drop is an optical effect due to the strong refraction of light rays at grazing incidence. Similar darkening at the bubble periphery can also be seen. It is remarkable that in none of the early 500 photographs taken in this set of experiments has ever more than one bubble been seen within a vapour-exploding drop. As is apparent from figure 3 , the bubbles form at random asymmetric locations within the drops, always rather close to the drop boundary (cf. $\$ 2$ ).

Of particular note is the somewhat regular pattern seen on the image of the bubbles in figure 3. It is hypothesized that this pattern is caused by variations of transmitted light through a regularly wrinkled liquid-vapour interface. At slightly later times 


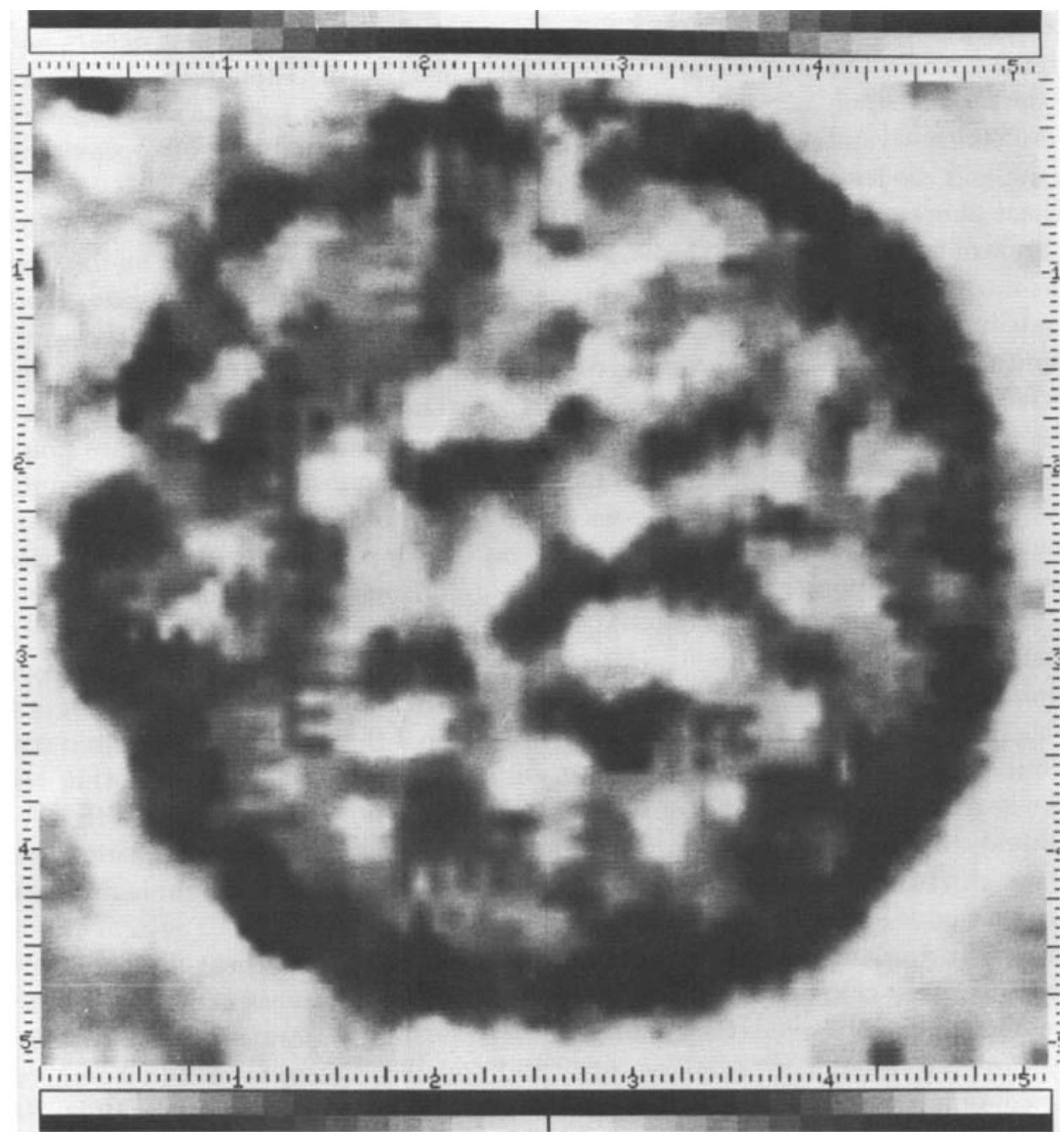

Figure 5. Image-cnhanced photograph of a bubble at $t=12 \mu \mathrm{s}$.

(figure 4) the bubble surface is extremely rough on a very small scale. When viewed in profile, the distortion is seen to be random and of large amplitude. This behaviour is quite different from that observed on vapour bubbles forming in boiling liquids at lower superheats, where the bubble surface is usually glassy smooth. This observation is the most important result from the present research. Another feature seen in the photographs of figures 3 and 4 is a bulbous protrusion of the bubble into the host fluid as soon as it has contacted the boundary of the drop (cf. §3.2).

The regular pattern on the bubble surface observed in figure 3 has been examined in more detail in one case by enhancing the image of the bubble by computer. The original photographic negative was digitized and was processed numerically using linear interpolation to expand the data base, median filtering to sharpen the edges, high-pass filtering to eliminate large-scale variations of photographic density, and contrast enhancement. $\uparrow$ The results are shown in figure 5. The enhanced image shows

$\dagger$ The authors aro indebted to Dr B. White and Mr D. Madura of the Medical Image Analysis Facility, Jet Propulsion Laboratory, for their aid in this effort. 
that the pattern covers the entire bubble and, in particular, that it occurs at the periphery where the unprocessed version appears uniform. Though the regularity of the pattern is much more striking in the enhanced image, there is still substantial asymmetry and randomness. The bubble in this figure has a diameter of approximately $150 \mu \mathrm{m}$ and the length scale of the pattern is $20-40 \mu \mathrm{m}$.

These photographs suggest that the extremely large evaporation rates occurring at the limit of superheat during the earliest stages of bubble growth drive an instability of the interface, a phenomenon that apparently has not previously been observed. The overall impression obtained from the photographs of figures 3-5 and those to be presented later is that, after the initial development, the wrinkling of the liquid-vapour interface saturates and persists at a nearly constant amplitude for the remainder of the evaporation process. The persistent roughening of the interface appears random in orientation and occurs on many length scales. The significance of the roughening to the dynamics of the evaporation process lies in a significant increase of the evaporating surface. The increase of the area of the randomly distorted surface yields a proportional increase in the evaporative mass flux, a process analogous to those which occur in other fluid-dynamic instabilities such as, for example, the vastly greater effectiveness of turbulent over laminar mixing. Unfortunately, because of the large difference between the index of refraction of liquid and vapour, it is impossible to view the interior of the bubble during the time that the evaporative surface is the roughest, so the fluid inside the bubble cannot be examined. Therefore, one can only speculate whether there might be a substantial amount of liquid within the bubble which has been torn from the liquid-vapour interface during the time when the mass flux across the interface is the largest. However, an estimate of the density of the fluid in the bubble (cf. \$3.1.3) indicates that some process like this may actually occur.

No observations of growing vapour bubbles at other than superheat-limit conditions have shown unstable evaporating surfaces, though disturbances caused by external sources have been observed (Hickman 1972) and possible evaporative instabilities have been proposed (Palmer 1976; Miller 1973). Bubbles observed so far in which vaporization occurs have had smooth and regular surfaces (Dergarabedian 1953, 1960; Florschuetz, Henry \& Khan 1969; Hooper, Eidlitz \& Faucher, 1970; Hewitt \& Parker 1968; Kosky 1968; Niino, Toda \& Egusa 1973). In those experiments the superheat was relatively low $\left(3 \cdot 6-36^{\circ} \mathrm{C}\right)$, while in the present experiments it is $105^{\circ} \mathrm{C}$, so the evaporative mass flux is much larger. Therefore, it is not surprising that new phenomena should appear, and that the results of experiments at the superheat limit might not be predicted by extrapolation from experiments at low superheat or from theories developed for near-equilibrium evaporation. Previous experiments at the superheat limit (Apfel \& Harbison 1975; Avedisian 1981) had inadequate spatial and time resolution to resolve the effects reported in this section. Actually, the behaviour at the liquid-vapour interface observed in the present experiments seems to be most similar to that seen during the flashing of free liquid surfaces by Grolmes \& Fauske (1974).

3.1.2. Pressures. Figure 6 shows representative pressure traces obtained during the first $50 \mu$ s of the explosion of three different drops of about the same diameter $(\sim 1 \mathrm{~mm})$. For these drops $50 \mu \mathrm{s}$ is about the time required for the liquid butane to completely evaporate from the drop, so it is the time at which the pressure reaches a maximum value. These traces exhibit a two-step increase of pressure at the beginning of the explosion that is characteristic of all the pressure traces obtained in these experiments. 


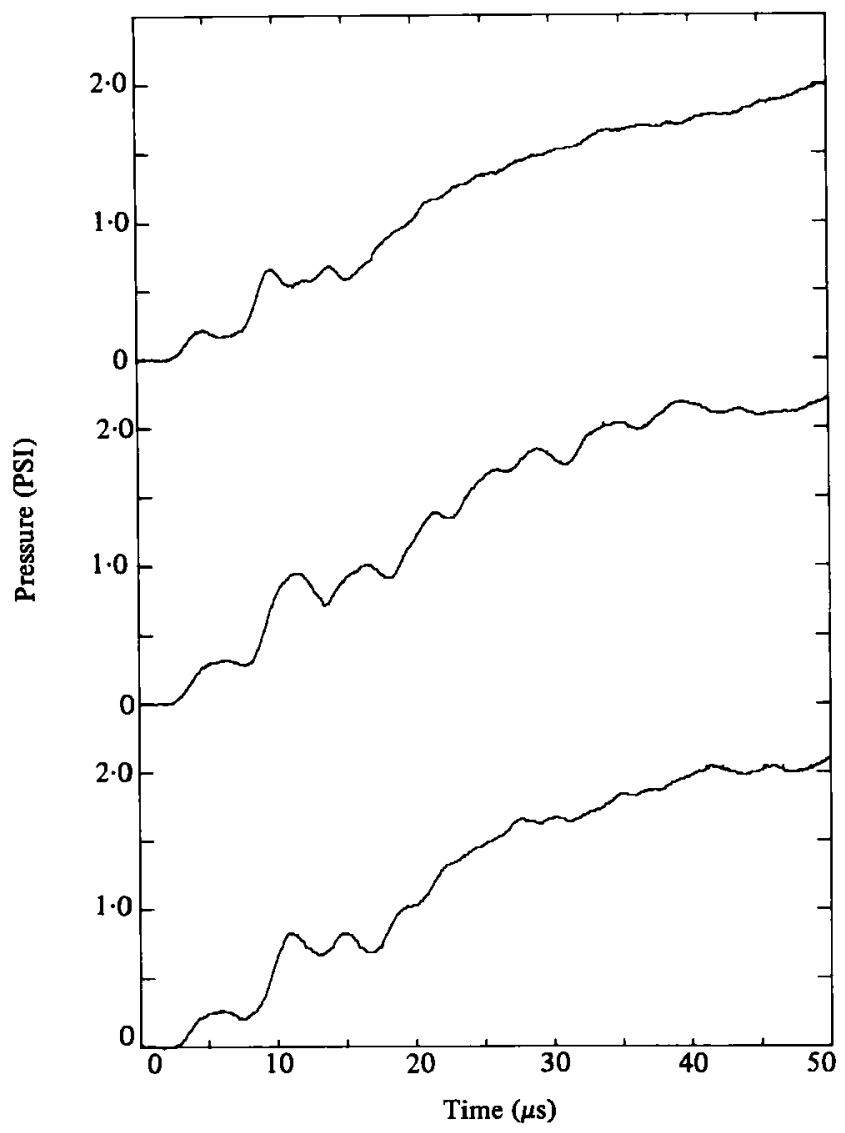

Fradre 6. The far-field pressure signal during the first $50 \mu \mathrm{s}$ of evaporation for 3 different explosions.

The rise time of the steps is of order $1 \mu \mathrm{s}$, the rise time of the pressure transducer, and their duration is approximately $5 \mu \mathrm{s}$. In view of the fact that the acoustic impedance of ethylene glycol is more than eight times greater than that of butane (table 1 ), $\dagger$ it is very likely that the blast waves generated during the initial instants of the explosion reverberate for some time within the drop. The fact that the round-trip acoustic transit time in a $1 \mathrm{~mm}$ diameter butane drop is equal to the $5 \mu \mathrm{s}$ spacing of the pulses observed at the beginning of the traces in figure 6 lends strong support to this hypothesis. While the drop is reverberating, waves normally incident upon the butaneethylene-glycol interface from within transmit to the exterior with pressure amplitude almost twice that of the incident amplitude, while obliquely incident waves transmit with somewhat smaller amplitude. Furthermore, the observed shape of the waveform during the initial instants and the directionality of the radiated field may be greatly affected by the lensing effect of the butane drop on the acoustic signals emanating from within it. At later times, when the acoustic wavelength of interest is larger than the drop diameter, the acoustic-impedance mismatch plays a decreasing role.

$\dagger$ A remarkably strong dependence of sound speed in liquid butane on pressure and temperature near the critical point (where the sound speed dips to only $130 \mathrm{~m} / \mathrm{s}$ ) leads, upon extrapolation of existing data near $105^{\circ} \mathrm{C}$ (Ribaud 1958), to the conclusion that the sound speed is as low as $410 \mathrm{~m} / \mathrm{s}$ at the superheat limit. 
3.1.3. Evaporative mass flux. It is important that the rate of change of the bubble radius $R$ is not the same as the velocity of the exterior liquid. In fact, the difference between these two quantities is just the evaporative flux across the liquid-vapour interface. The evaporative flux does not appear explicitly in theories that assume the vapour phase to be in thermodynamic equilibrium, yet it is the fundamental parameter that characterizes the behaviour and severity of vapour explosions. Using the data obtained in these experiments, an order-of-magnitude estimate of the evaporative flux has been calculated by inserting the photographically determined bubble growth rate and the acoustic source strength obtained from the pressure measurements into the global conservation equation for mass in the bubble.

$$
\frac{d M}{d t}=\rho_{\ell} \frac{d V}{d t}-\rho_{\ell} Q
$$

where $M$ is the total mass in the bubble, $\rho_{\ell}$ is the liquid density, $V$ is the volume of the bubble and $Q$ is the acoustic source strength, that is, the rate of volume outflow in the fluid outside the bubble. The effective evaporative mass flux $\dot{m}$ is calculated from $d M / d t$ by dividing by the measured mean evaporating area $A$ :

$$
\dot{m}=\frac{1}{A} \frac{d M}{d t}
$$

Unfortunately, the data obtained so far are not sufficient to provide a precise measurement of the bubble growth rate, because only one picture is obtained from each explosion. In order to estimate the rate of change of bubble volume it is necessary to measure the bubble diameter in pictures that have been obtained during different explosions. Variable bubble shape from run to run, caused by the random location at which the bubbles form in the drops, introduces inaccuracy into this calculation. However, the fact that every bubble is symmetric about an axis passing through the original point of nucleation in the drop and the point at which the bubble first contacts the host liquid (cf. $\$ 3.2$ and figure 10) provides the basis for a simple geometrical model of the bubble shape. The shape of 22 bubbles in representative photographs at times ranging from $8 \cdot 3$ to $91 \mu$ s was digitized, and the bubble volume and mean evaporating surface area were calculated, treating the bubbles as bodies of revolution. In fact, only those bubbles whose axis of symmetry was in or near the plane of the photograph were analysed. The bubble volume $V$ and the mean evaporating surface area $A$ were derived from the co-ordinates of the outline of the bubble in the photograph. For $A$ only the area of the bubble surface estimated to be in contact with the liquid butane was used. No correction for the roughness of the interface was made. The data for $V$ and $A$ were fit to power laws in time for use in (2) and (3). The effective radius of a spherical bubble implied by the volume data is plotted versus time in figure 7 . It is remarkable that the growth rate appears to be linear, with a mean velocity of approximately $14.3 \mathrm{~m} / \mathrm{s}$. Since the present data are obtained after onset of instability, it is not surprising that the growth rate disagrees with the predictions of the classical diffusionlimited theory. Indeed, the observed rate is substantially less than the linear growth rate (dashed line, $40 \mathrm{~m} / \mathrm{s}$ ) predicted by the theory for the inertially dominated stage at very small times, but is larger than the prediction (solid line) for later times during the actual period of observation in these experiments, when the limiting effects of heat transfer are supposed to dominate. As will be seen below, the fact that the observed 


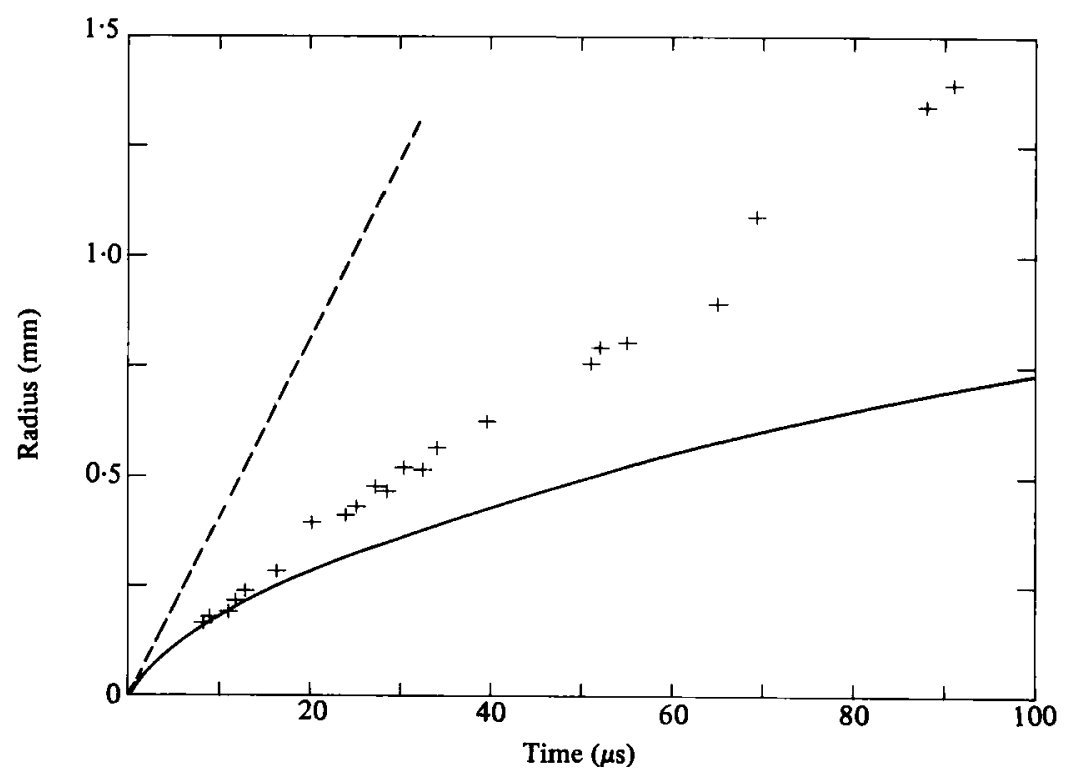

FIgURE 7. Effective bubble radius as a function of time: + , experiment; - - , classical theory; ----- , classical inertial growth rate, $40 \mathrm{~m} / \mathrm{s}$.

growth rate is less than the predicted inertially dominated rate does not mean that evaporation rates are particularly small. Since both the bubble growth rate and the evaporative mass flux contribute to the effect of the vapour explosion on the surrounding fluid, it is important that both quantities be accurately accounted for.

Three simplifying assumptions are made in the calculation of the equivalent acoustic source strength $Q$ of the explosion. First, the growing bubble is treated as a compact source, that is, $\lambda \gg d$, where $\lambda$ is the wavelength of the radiated sound and $d$ is the diameter of the bubble or the drop. Within one wavelength of a source, the motion is not wavelike, so, in this case, the acoustic-impedance mismatch between the drop and host liquids plays no role. It is clear that this assumption is not valid at small times, when pressure waves reverberate within the drop (cf. §3.1.2). Indeed, at these early times the method used here probably overestimates the acoustic source strength, and thus underestimates the evaporation rate $\dot{m}$ for early times. However, for times greater than, say, 40-80 $\mu \mathrm{s}$, when reverberations have died out and acoustic wavelengths are relatively long, the present estimate should be valid. Secondly, it is assumed that the pressure transducer is mounted in the far field of the bubble $(r \gg d$, where $r$ is the bubble-to-transducer distance). Finally, the source is assumed to be spherically symmetric. Though, as shown by the photographs, the source is actually axisymmetric with the axis of symmetry randomly oriented from explosion to explosion, for the purpose of this estimate the effects of non-sphericity are minimized by averaging over several runs (cf. below). Then, the source strength can be calculated from

$$
Q(t)=\frac{4 \pi r}{(1+\alpha) \rho_{\infty}} \int_{0}^{t} p\left(t^{\prime}+r / a\right) d t^{\prime}
$$

where $\alpha$ is the coefficient of reflection of waves normally incident on the transducer face (taken to be 0.83 for an aluminium-glycol interface), and $p, \rho_{\infty}$ and $a$ are the pressure measured by the transducer, the host liquid density and sound speed, 


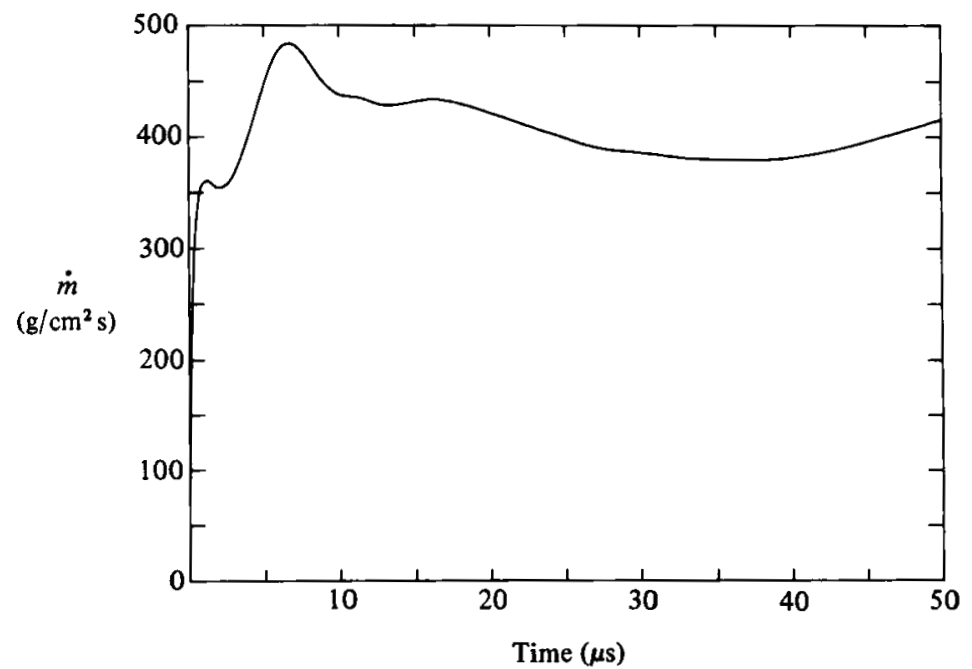

Figure 8. Average evaporative mass flux.

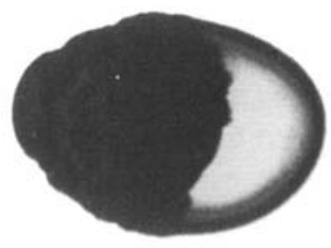

(a)

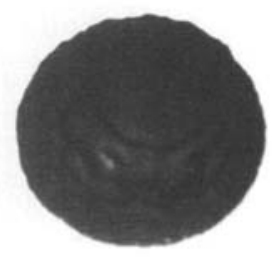

(d)

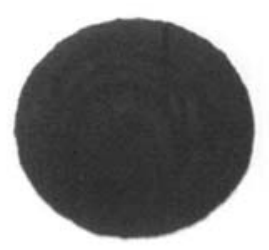

(b)

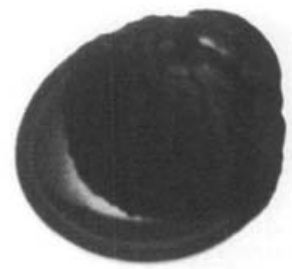

(c)

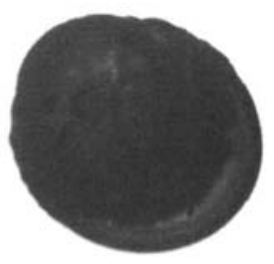

(e)

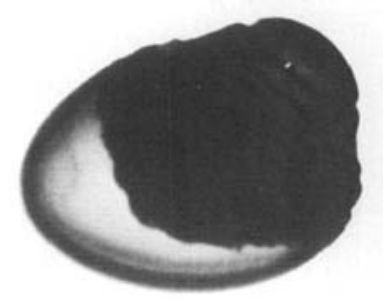

$(f)$

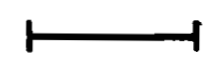

Figure 9. Fully developed bubbles, showing roughened evaporating surface and circumferential waves (55-65 $\mu \mathrm{s}$ ). Scale indicates $1 \mathrm{~mm}$. 
respectively. A detailed pressure trace is obtained for the entire duration of each explosion in these experiments. In order to form an estimate representative of a typical vapour explosion and consistent with the composite explosion built up by using photographs from different explosions to determine bubble radius, seven pressure signals were averaged for the computation of an average source strength from (4). It is interesting that the characteristic two-step initial rise seen in figure 6 survives the averaging with little change, but the subsequent oscillations, which are not repeatable from run to run, are very strongly smoothed by the averaging.

The effective evaporative mass flux calculated from (2) and (3) is shown in figure 8. The mass flux rises very rapidly, and after some fluctuations levels off at about $400 \mathrm{~g} /$ $\mathrm{cm}^{2} \mathrm{~s}$. This value is to be compared with mass fluxes typical of evaporation at low superheat, which are of the order of $10^{-3}-10^{-1} \mathrm{~g} / \mathrm{cm}^{2} \mathrm{~s}$. Only in extreme situations such as vacuum distillation (Hickman 1972) or laser ablation (Ready 1965) does the mass flux reach the magnitude observed in these experiments. Indeed, this mass flux is of the same order as the maximum possible flux across a smooth interface given by kinetic theory, $\frac{1}{4} n \bar{c}$, despite the fact that, as will be shown next, the density inside the bubble is of the order of liquid density!

An estimate of the mean density $\rho_{\text {eff }}$ within the bubble has been calculated by dividing $M(t)$ by $V(t)$. A plot of $\rho_{\text {eff }}$ versus time shows that, after the transients, it settles down to a nearly constant value, decreasing from $0.2 \mathrm{~g} / \mathrm{cm}^{3}$ at $10 \mu \mathrm{s}$ to $0.13 \mathrm{~g} / \mathrm{cm}^{3}$ at $50 \mu \mathrm{s}$. These values are more than one half of the critical density of butane, $0 \cdot 23 \mathrm{~g} / \mathrm{cm}^{3}$ ! A possible explanation of this seemingly anomalous result is obtained by considering a steady-state model of evaporation (cf. $\$ 4.2$ ).

Comparison of experimental results with the predictions of the classical theory (e.g. in figure 7) was made using the approximate analytical expression for $R$ suggested by Mikic, Rohsenow \& Griffith (1970), rather than computing the exact solutions of Prosperetti \& Plesset (1978). However, the modified method for evaluating the vapour pressure suggested by Theofanous \& Patel (1976) and Prosperetti \& Plesset was used. Taking the predicted evaporative mass flux $\dot{m}$ to be

$$
\dot{m}=\rho_{\mathrm{v}}\left(T_{\ell}\right) \frac{d R}{d t},
$$

where, in accordance with the assumptions of the classical theory, the relevant density is taken to be $\rho_{v}\left(T_{f}\right)$, the density of saturated vapour at the temperature $T_{\ell}$ of the liquid interface, the value of $\dot{m}$ predicted at $10 \mu$ s for the conditions of the present experiments is two orders of magnitude less than observed, and becomes even smaller with increasing time.

\subsection{Jet formation}

In figures 3 and 4 a characteristic bulging into the host fluid is observed on all bubbles which have grown large enough to contact the boundary of the drop. Figure 9 shows photographs of typical drops at later times. These photographs show how the single bulge seen in the previous figures develops into a unique axisymmetric structure consisting of circumferential waves culminating in a spherical cap. The axis of symmetry of this structure passes through the original drop centre, the centroid of the bubble and the point where the bubble surface first contacts the host. Random orientation of this axis relative to the plane of the photograph due to the random nature of nucleation results in a variety of perspectives of the developing structure. The image 


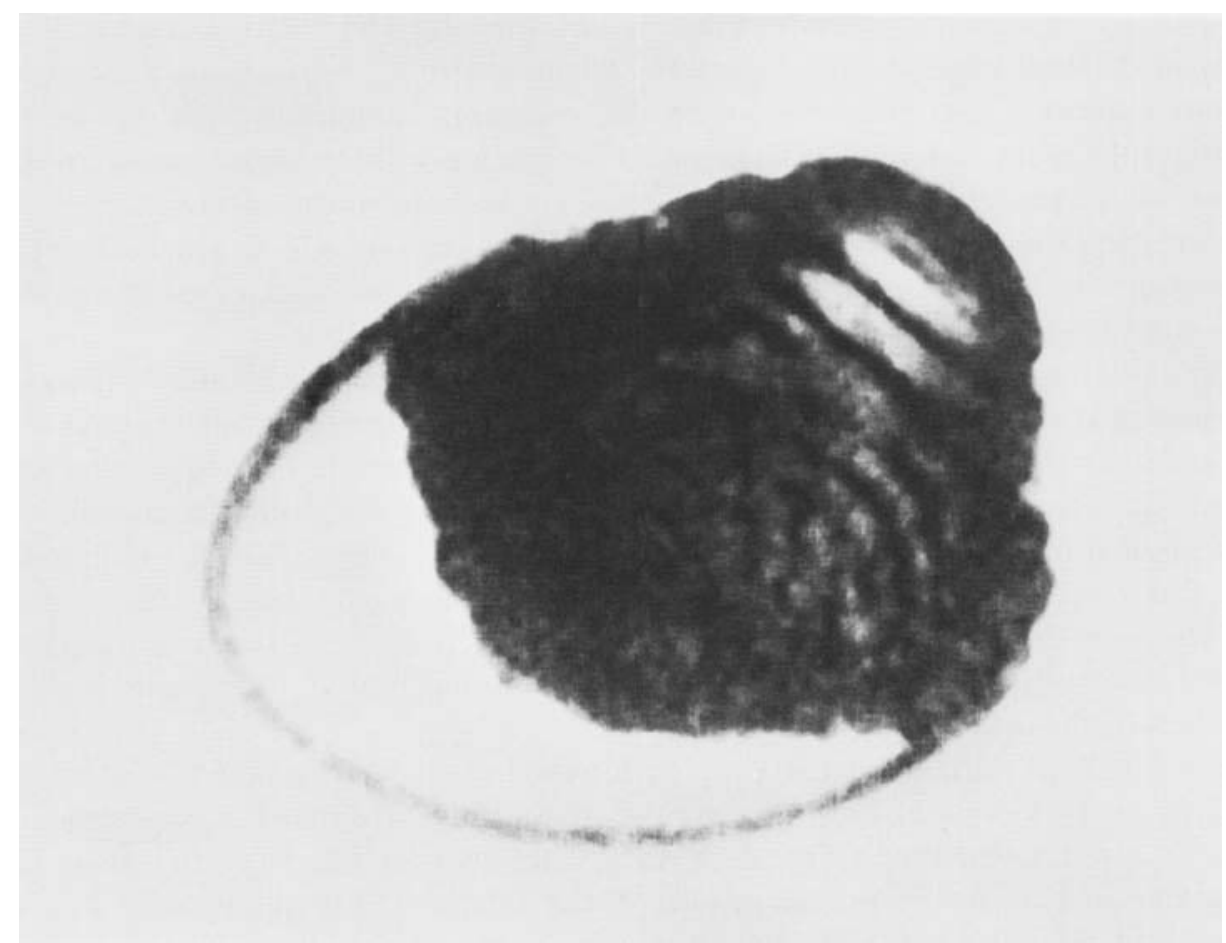

Figure 10. Contrast-enhanced image of the bubble of figure $9(f)$.

of figure $9(f)$ is shown in figure 10 magnified and contrast-enhanced by computer to exhibit all of the features of this stage of the explosion. Peculiar bubble shapes and surface waves have been seen previously in studies of cavitation-bubble collapse (Ellis 1965) and of interacting bubbles (Hooper et al. 1970), but in the present context this phenomenon is completely unexpected and rather bizarre in appearance. It is conjectured that the protrusion into the host liquid and the surface distortions are both driven by a vapour jet from the opposing evaporating surface impinging on the host fluid. Preliminary experiments indicate that the formation of circumferential waves on a gas-liquid interface may be a general property of transient gas jets into liquids, and this question is being investigated further at this laboratory in a separate investigation. Also seen in figure 10 is the fact that the random roughening of the liquidvapour interface caused by rapid evaporation persists on that portion of the surface which contacts the host liquid, that is, even after the evaporation terminates. However, this roughness soon smooths out by wavespreading along the bubble surface (figure $11 a)$.

\subsection{Bubble oscillations}

Immediately after the liquid in the superheated drops is completely vaporized in these experiments, there is substantial overpressure in the bubble and a great deal of kinetic energy stored in the outward motion of the liquid surrounding the bubble. Therefore the growing bubble overshoots the equilibrium radius, at which the pressure in the bubble equals the ambient pressure, and begins to oscillate violently. As a consequence, during the vapour explosion of a single drop the far-field radiated pressure progresses through the following sequence. It first reaches a maximum when 

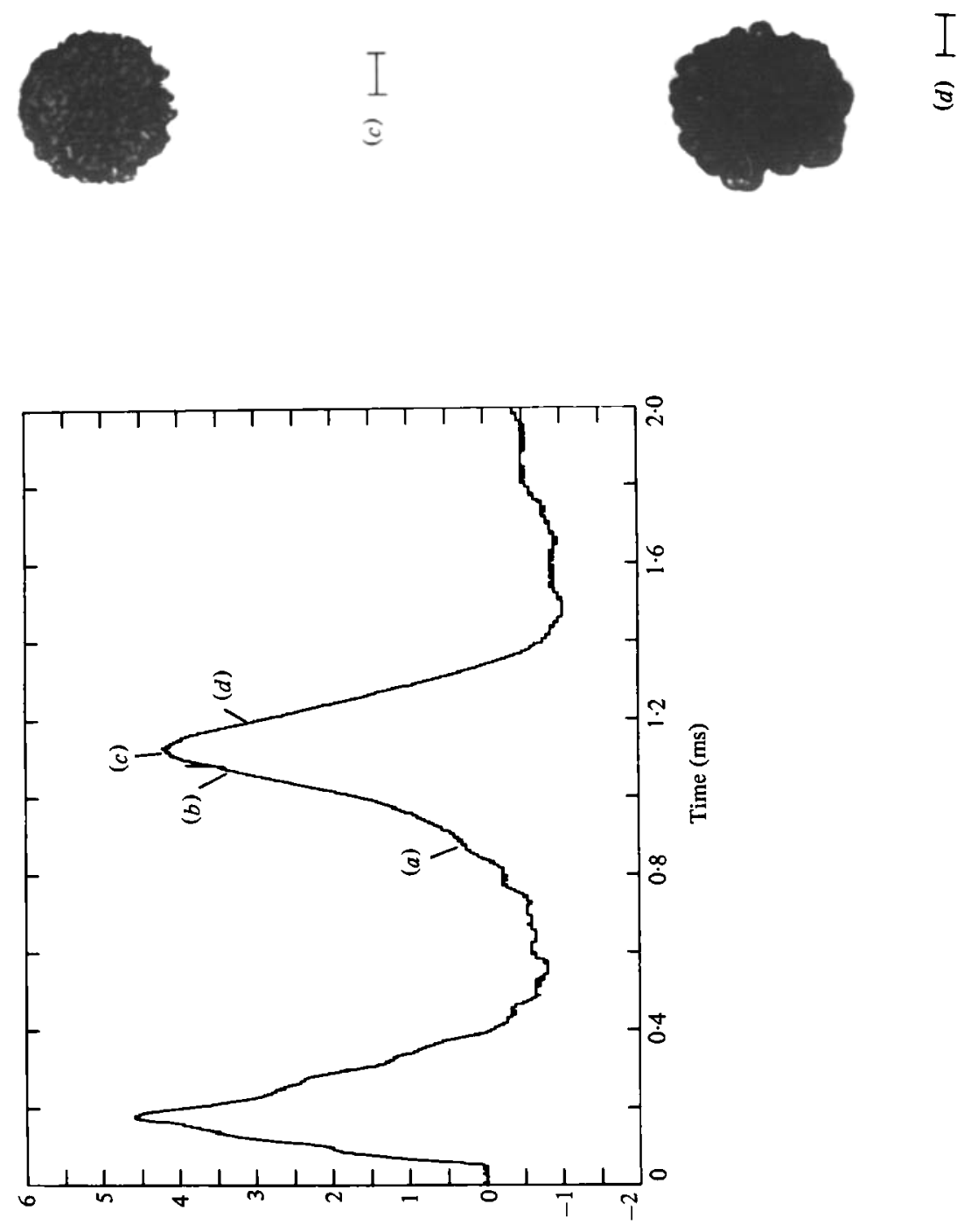

(ISd) annsserd

घี
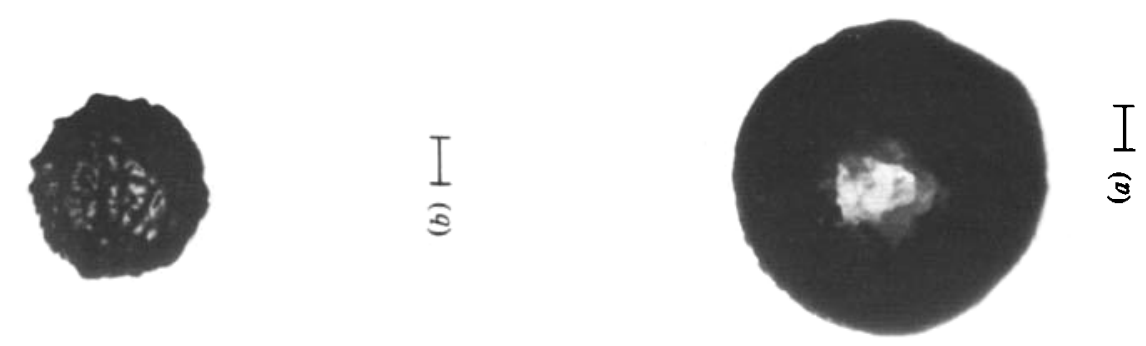
the vaporization of the superheated liquid stops. It then decreases to a minimum as the bubble decelerates outward to its maximum radius. Then, as the bubble collapses the radiated pressure increases, reaching a sharply peaked maximum when the bubble is at minimum radius (Cole 1948). Figure 11 shows a typical pressure history and four photographs of representative bubble shapes during the first collapse after explosion. As can be seen from the photographs, the bubbles are subject to the Rayleigh-Taylor instability (Taylor 1950) during this phase of the oscillation. The measured values of peak far-field radiated pressure and minimum bubble diameter $(2 \mathrm{~mm})$ imply that the outward acceleration at minimum radius is of order $10^{4} \mathrm{~g}$, so the occurrence of Rayleigh-Taylor instability is not surprising.

The general stability criteria of Birkhoff (1956) show that oscillating bubbles are unstable when

$$
\begin{aligned}
& \Gamma>0, \\
& \Gamma<0, \quad \frac{d}{d t}\left(R^{6} \Gamma\right)>0
\end{aligned}
$$

where

$$
\Gamma=(n-1)\left[\frac{\ddot{R}}{R}-\frac{(n+1)(n+2) \sigma}{\rho_{\infty} R^{3}}\right]
$$

is the acceleration parameter in the equation for the growth of the amplitude $a_{n}$ of the $n$th mode of the surface perturbation (Plesset \& Prosperetti 1977; Strube 1971),

$$
\ddot{a}_{n}+3 \frac{\dot{R}}{R} \ddot{a}_{n}-\Gamma a_{n}=0,
$$

and where $\sigma$ is the surface tension. Criterion $(6 b)$ is interesting because it predicts instability under circumstances in which a plane interface would be stable. In this case the surface perturbation oscillates in time with algebraically increasing amplitude. On the other hand, when $\Gamma>0$ the instability grows monotonically and exponentially, as in the plane case. It is the latter behaviour that dominates in the present experiments. For the higher modes the most-unstable wavenumber $k^{*}$ is the same as for a plane interface:

$$
k^{*}=\left(3 \rho_{\infty} \ddot{R} / \sigma\right)^{\frac{1}{2}} .
$$

With values appropriate to the present experiments this wavenumber agrees within a factor of two with that observed in figure $11(c)$.

As the bubble continues to oscillate, the distortions which developed during the first collapse (figure 11) grow continuously larger until the bubble disintegrates into a cloud of smaller bubbles.

\section{Discussion}

The most important results of the present experiments are (i) the observation of the wrinkling of the liquid-vapour interface during the early growth stage of bubbles in vapour-exploding butane and (ii) the estimate showing that the evaporative fluxes after onset of the instability are extremely large. In this section we discuss a proposed mechanism for the instability and point out the consequences of the observed behaviour of the vaporization process after onset of the instability. 


\subsection{The Landau instability}

It is proposed that the distortion and roughening of the liquid-vapour interface observed in the present experiments is attributable to an inertial instability first introduced by Landau (1944; cf. also Landau \& Lifshitz 1959) to explain the instability of laminar flames $\dagger$ and subsequently discussed, together with several other mechanisms, in connection with evaporation by, for example, Miller (1973) and Palmer (1976). The important physical processes influencing stability that are included in the theory are mass flux across the liquid-vapour interface, acceleration of the fluid normal to the interface and surface tension. The Landau instability has been treated for spherical flames growing at constant speed by Istratov \& Librovich (1969), but the effects of acceleration and surface tension have not yet been included in an analysis applicable to rapid evaporation in spherical systems. Acceleration may be destabilizing to growing bubbles only during the initial surface-tension-controlled stage of growth, when the acceleration is outward. However, for evaporation at the superheat limit, according to the classical theory of bubble growth, the surface-tension-controlled stage is extremely brief, lasting only $\sim 10^{-10} \mathrm{~s}$ for the conditions of the present experiments. Though the outward accelerations during this time are enormous $\left(\sim 10^{9} \mathrm{~g}\right)$ the radii are very small, so $\Gamma<0$ in (7), and bubbles are Rayleigh-Taylor stable even during this stage. On observable time scales (say, a few microseconds) the growth before the onset of instability is diffusion-limited, and the consequent deceleration of the fluid motion, which for the conditions of the present experiments is of order $10^{5} \mathrm{~g}$, is stabilizing.

Thus it must be the mass flux across the liquid-vapour interface that drives the evaporative instability observed in the present experiments. This instability has not been observed before in other evaporative systems because the rates of evaporation in those cases were orders of magnitude smaller than those which occur at the superheat limit. The presence of substantial mass flux across a distorted liquid-vapour interface has the important consequence that, though the incoming flow upstream of the discontinuity (i.e., in the liquid phase) may be irrotational, vorticity is generated by the flow transition at the interface and appears in the downstream flow (the vapour phase). It is this interaction that drives the Landau instability and that distinguishes it from other instabilities.

In order to weigh the feasibility that the Landau mechanism of instability causes the interface roughening observed in the present experiments, calculations have been made of the stability limits and growth rates given by the dispersion relation of the Landau theory, using the mass flux and acceleration predicted as a function of time by the classical theory of bubble growth (cf. §3.1.3). It is found that for small times $\left(t<10^{-10} \mathrm{~s}\right)$, as described above, the accelerations stabilize the interface, and that for large times $\left(t>10^{-6} \mathrm{~s}\right.$ for conditions of the present experiments) a classically growing bubble would again become stable because the evaporative mass flux decreases below a critical value, but at intermediate times the liquid-vapour interface of butane exploding at the superheat limit is indeed predicted to be unstable over a large range of wavenumbers $\left(5 \times 10^{3}<k<1.3 \times 10^{5} \mathrm{~cm}^{-1}\right)$. However, in a spherical system another limitation on instability arises from the fact that disturbances of wavelength greater than the diameter of the bubble cannot exist. Therefore, for the purpose of these calculations we have imposed an ad hoc low-wavenumber limit of instability for

$\dagger$ The authors are indebted to Professor F. E. Marble of the Jet Propulsion Center for suggesting that the Landau instability might be relevant to our experiment. 


\begin{tabular}{llll} 
& \multicolumn{1}{c}{$\mathrm{C}_{4} \mathrm{H}_{10}$} & \multicolumn{1}{c}{$\mathrm{H}_{2} \mathrm{O}$} & $\mathrm{Na}$ \\
$\omega\left(\mathrm{s}^{-1}\right)$ & $1.2 \times 10^{7}$ & $2.3 \times 10^{7}$ & $3.7 \times 10^{8}$ \\
$\tau(\mathrm{s})$ & $0.25 \times 10^{-6}$ & $1.3 \times 10^{-6}$ & $8.0 \times 10^{-6}$ \\
$F$ & 2.9 & 29 & 2900
\end{tabular}

TABLE 2. Growth rate, duration of instability, and their product

spherical bubbles, $k=2 \pi / R$. Actually, as shown by Istratov \& Librovich (1969), since disturbances on growing bubbles must grow more rapidly than the bubble itself before the behaviour can be termed unstable, a finite number of the lowest modes of oscillation of the bubble may be stable. Furthermore, spherical expansion of the perturbed system leads in some cases to slower, algebraic, growth of instabilities rather than the exponential growth predicted for planar systems. Therefore, expanding bubbles may be somewhat more stable than implied by the estimate obtained here, but, in view of the uncertainty of the values of the parameters used in these calculations, the added complexity of a more sophisticated criterion for the low-wavenumber limit of instability does not seem justified. Application of this limit to butane at the superheat limit restricts the range of wavenumbers at which instability can occur to $6 \times 10^{3}<k<$ $9 \times 10^{4} \mathrm{~cm}^{-1}$. The locus of wavenumbers at which the maximum growth rate of disturbances on a plane interface occurs happens to fall within this band of wavenumbers. We adopt these maximum growth rates as an estimate of the upper bound of the growth rate of disturbances on spherical bubbles.

The scale of the disturbances observed on bubbles at the earliest times in the present experiments (figure 5) is 2-4 times larger than the largest scale in the unstable band of wavenumbers cited above, but the observations are made at times an order of magnitude larger than those during which the linear theory predicts the bubbles to be unstable. Thus these experiments are not carried out under conditions that would provide definite verification that the Landau mechanism is indeed the one that drives the instability, but the results are consistent with the Landau mechanism, providing the scale of the disturbances that develop during the linear phase of the instability at very small times remains frozen in the flow during the initial development of the nonlinear stage. Further experiments should be carried out to investigate this possibility.

A simple quantitative measure of the relative susceptibility to this evaporative instability of a substance boiling at its superheat limit can be obtained from the results of the calculations described in this section by, for example, multiplying a typical value $\omega$ of the maximum grow th rate calculated to occur within the region of instability by the time interval $\tau$ during which a classically growing bubble is predicted to be linearly unstable. The resulting figure of merit $F$ is the amount by which disturbance amplitudes are exponentiated during instability, according to linear stability theory for plane interfaces. We take for $\omega$ the largest value of maximum grow th rate calculated within the region of instability, which, incidentally, always occurs near the beginning of the period of instability. The predicted values of $\omega, \tau$ and $F$ for butane at the superheat limit are given in table 2 . The result $F=2.9$ indicates that the instability of butane is marginal, and that the discovery of this phenomenon by studying the vapour explosion of butane droplets was a matter of some luck!

The susceptibility of other substances to instability can be estimated by inserting 
the appropriate physical properties into the above calculations. This has been done for two technically important fluids, water and liquid sodium, though the properties and behaviour of sodium at the limit of superheat are not well known, so that estimate is very crude. The results listed in table 2 show that these two fluids are, indeed, very much more unstable than butane.

\subsection{Empirical model of evaporation after onset of instability}

The results presented in figures 6-8 indicate that after the evaporative instability becomes nonlinear and saturated the evaporation process seems to be quasi-steady, with the bubble radius increasing at constant speed, say $\dot{R}$, the far-field pressure increasing at a roughly linear rate $\overline{d p / d t}$, and the evaporative flux being a constant $\dot{m}_{0}$. It is interesting to inquire what the consequences of these simplifications are. Another parameter that must be defined to incorporate the geometry of a growing bubble properly within a single droplet of volatile liquid is the fraction $\beta$ of the bubble surface across which evaporation actually takes place, the remainder, $1-\beta$, being the fraction of the bubble's surface in contact with the non-evaporating host fluid. Calculations of $\beta$ from the data of the present experiments suggests that, for the purposes of this model, $\beta \sim 0 \cdot 5$. With $\beta, \dot{m}_{0}$ and $\dot{R}$ assumed constant, the following results are obtained.

(i) The bubble volume $V$ and the total mass $M$ within the bubble increase as $t^{3}$ :

$$
\begin{gathered}
V=\frac{4}{3} \pi \overline{\dot{R}}^{3} t^{3}, \\
M=\frac{4}{3} \pi \dot{R}^{2} t^{3} \beta \dot{m}_{0} .
\end{gathered}
$$

(ii) The effective vapour density within the bubble $\rho_{\text {eff }}$ is constant:

$$
\rho_{\text {err }}=\beta \frac{\dot{m}_{0}}{\dot{R}} .
$$

(iii) The time $t_{0}$ for liquid initially contained within a drop of radius $R_{0}$ to completely boil is proportional to $R_{0}$ :

$$
t_{0}=R_{0}\left[\beta \frac{\dot{m}_{0}}{\rho_{1}} \overline{\dot{R}}^{2}\right]^{-\frac{1}{3}} \text {. }
$$

(iv) The far-field pressure increases linearly with time:

$$
\frac{\overline{d p}}{d t}=\frac{2 \rho_{\infty} \overline{\dot{R}}^{2}}{\tau}\left[\overline{\dot{R}}-\beta \frac{\dot{m}_{0}}{\rho_{1}}\right] .
$$

(v) The maximum far-field pressure at the end of the evaporative stage is proportional to $t_{0}$, and therefore to $R_{0}$ :

$$
p_{\max }=\frac{\overline{d p}}{d t} t_{0}
$$

These equations are not only all in qualitative agreement with the observations, but, if data already cited in this paper are inserted into the right-hand sides of (12)-(14), the resulting numbers agree with results independently derived from the measurements. Namely,

$$
\begin{gathered}
\rho_{\text {eff }}=0.142 \mathrm{~g} / \mathrm{cm}^{3}, \\
t_{0}=10^{2} R_{0} \quad\left(t_{0} \text { in } \mu \mathrm{s}, R_{0} \text { in } \mathrm{mm}\right) \\
\overline{d p} / d t=1.4 \times 10^{3} \mathrm{bar} / \mathrm{s} .
\end{gathered}
$$


These results show that the measurements made during the course of these experiments are self-consistent. The reason for the rather simple behaviour exhibited by the vaporization process during the nonlinear saturated stage is not understood.

If, further, the fraction of the fluid in the bubble that must be in the liquid state is calculated by invoking the conservation of energy in the flow across the liquid-vapour interface (using the Rayleigh equation to determine the vapour pressure), it is found that, remarkably, the liquid content is $37 \%$ by mass.

\section{Conclusions}

An interfacial instability driven by rapid evaporation has been observed on the surface of single, rapidly growing bubbles in droplets of liquid butane boiling at the superheat limit. The instability is observed during relatively early stages of its development, when the pattern of the disturbances on the bubble is relatively regular, and during the later stages, when the wrinkling of the surface saturates at nearly constant amplitude, is random and occurs on many length scales. The rate of evaporation is estimated from the data to be two orders of magnitude greater than would be predicted without accounting for the effects of instability. An estimate of the mean density within the bubbles during the evaporative stage indicates that it is more than one half of the critical density of butane.

It is proposed that the theory developed by Landau for explaining the instability of laminar flames may be applicable to the evaporating systems studied in this work. The experimental observations are consistent with this hypothesis, so long as the disturbances which develop during the linear stage of the instability (at times predicted to be much smaller than are observable in the experiments) remain frozen in the flow during the early part of the nonlinear phase of the instability. The disturbance growth rate and the time interval during which the bubbles are predicted to be linearly unstable imply that butane is marginally unstable, but that other technically important liquids (water and sodium) may be more unstable.

An interesting dynamical effect has been observed on the surface of bubbles that have grown large enough to contact the non-evaporating host liquid surrounding the boiling drop. A series of toroidal waves terminating in a spherical cap form on the vapour-host-liquid interface, presumably owing to the effects of the vapour jet generated at the evaporating interface impinging on the host-liquid interface.

After all of the volatile liquid in the drop is vaporized, the bubble overshoots the equilibrium configuration and begins to oscillate violently. Outward acceleration of the liquid-vapour interface while the bubble is at minimum radius is of order $10^{4} \mathrm{~g}$, so the bubble is severely Rayleigh-Taylor unstable and rapidly breaks up into a cloud of tiny bubbles.

This research was supported by the United States Department of Energy, Division of Chemical Sciences, under Project Agreement DE-AT03-80ER10634.

\section{REFERENCES}

Altenburg, K. 1952 Die temperaturabhangigheit der ultraschallgeschwindigkeit und der oberflachenspannung des athylenglykols. Z. physik. Chem. 201, 91 .

Apfel, R. E. \& Harbison, J. P. 1975 Acoustically induced explosions of superheated droplets. J. Acoust. Soc. Am. 57, 1371. 
Anderson, R. P. \& Armstrong, D. R. 1974 Comparison between vapor explosion models and recent experimental results. A.I.Ch.E. Symp. Series, 70 (138), 31.

Avedisian, C. T. 1981 Effect of pressure on bubble growth within droplets heated to their limits of superheat. A.S.M.E. Paper no. 81-HT.11.

Birkhoff, G. 1956 Stability of spherical bubbles. Q. Appl. Math. 13, 451.

Board, S. J. \& Caldarola, L. 1977 Fuel-coolant interactions in fast reactors. In Symp. on Thermal and Hydraulic Aspects of Nuclear Reactor Safety, Vol. 2: Liquid Metal Fast Breeder Reactions (ed. O. C. Jones \& S. G. Bankoff), p. 195. A.S.M.E.

Butvid, M. G. \& Sussman, M. V. 1978 Superheated liquids containing suspended particles. Nature 275, 203.

Cole, R. H. 1948 Underwater explosions. Princeton University Press.

Colgate, S. A. \& Siqurgeirson, T. 1973 Dynamic mixing of water and lava. Nature 244, 52.

Dergarabedian, P. 1953 The rate of growth of vapor bubbles in superheated water. Trans. A.S.M.E. E, J. Appl. Mech. 20, 537.

Dergarabedian, P. 1960 Observations on bubble growth in various superheated liquids. J. Fluid Mech. 9, 40.

Dufour, L. 1861 a Sur l'ebullition des liquides. C.R. Acad. Sci. Paris 52, 986.

Dufour, L. $1861 b$ Sur l'ebullition des liquides. C.R. Acad. Sci. Paris 53, 846.

Ellis, A. T. 1965 Parameters affecting cavitation and some new methods for their study. California Inst. Tech. Hydrodyn. Lab. Rep. E-115.1.

Florshuetz, L. W., Henry, C. L. \& KhaN, A. R. 1969 Growth rates of free vapor bubbles in liquid at uniform superheats under normal and zero gravity conditions. Int. J. Heat Mass Transfer 12, 1465.

Gibson, R. E. \& Loeffler, O.H. 1941 Pressure-volume-temperature relations in solutions. $V$. The energy-volume coefficients of carbon tetrachloride, water and ethylene glycol. J. Am. Chem. Soc. 63, 898.

Grolmes, M. A. \& Fauske, H. K. 1974 Axial propagation of free surface boiling into superheated liquids in vertical tubes. In Proc. 5th Int. Heat Transfer Conf., Paper B1.7, 4, 30. Japan Soc. Mech. Engrs and Soc. Chem. Engng, Japan.

Hewitt, H. C. \& Parker, J. D. 1968 Bubble growth and collapse in liquid nitrogen. Trans. A.S.M.E. C., J. Heat Transfer 90, 22.

Hickman, K. 1972 Torpid phenomena and pump oils. J. Vac. Sci. Tech. 9, 960.

Hooper, F. C., Eidlitz, A. \& Faucher, G. 1970 Bubble growth and pressure relationships in the flashing of superheated water. Technical Publication no. 6904, vols 1-3. University of Toronto, Dept Mech. Engng.

Istratov, A. G. \& Librovich, V. B. 1969 On the stability of gasdynamic discontinuities associated with chemical reactions. The case of a spherical flame. Astron. Acta. 14, 453.

Kosky, P. G. 1968 Bubble growth measurements in uniformly superheated liquids. Chem. Engng Sci. 23, 695.

Landat, L. D. 1944 On the theory of slow combustion. Acta Physiochimica U.R.S.S. 19, 77.

Landau, L. D. \& Lifshitz, E. M. 1959 Fluid Mechanics, problem 2, p. 479. Pergamon.

Mikic, B. B., Rohsenow, W. M. \& Griffith, P. 1970 On bubble growth rates. Int. J. Heat Mass Transfer 13, 657.

Muller, C. A. 1973 Stability of moving surfaces in fluid systems with heat and mass transport - II. Combined effects of transport and density difference between phases. A.I.Ch.E.J. $19,909$.

Moore, G. R. 1959 Vaporization of superheated drops in liquids. A.I.Ch.E.J. 5, 458.

Nino, M., Topa, S. \& Equsa, T. 1973 Experimental investigation of nucleation and growth of a single bubble using laser beam heating. Heat Transfer Japanese Research 2, 26.

Palmer, H. J. 1976 The hydrodynamic stability of rapidly evaporating liquids at reduced pressure. J. Fluid Mech. 75, 487.

Plesset, M. S. \& Prosperetti, A. 1977 Bubble dynamics and cavitation. Ann. Rev. Fluid Mech. 9, 145.

Porteous, W. \& Blander, M. 1975 Limits of superheat and explosive boiling of light hydrocarbons and hydrocarbon mixtures. A.I.Ch.E.J. 21, 560. 
Prosperetti, A. \& Plesset, M. S. 1978 Vapour bubble growth in a superheated liquid. $J$. Fluid Mech. 85, 349.

Ready, J. F. 1965 Effects due to absorption of laser radiation. J. Appl. Phys. 36, 462.

REID, R. C. 1978 Superheated liquids: a laboratory curiosity and, possibly, an industrial curse. Chem. Engng Ed. 12, 60.

REID, R. C. 1979 Possible mechanism for pressurized-liquid tank explosions or BLEVE's. Science 203, 1263.

Rigaud, M. G. 1958 Variation de la vitesse des ultrasons dans le butane normal en fonction de la temperature et la pression. C.R. Acad. Sci. Paris 246, 2356.

SALLET, D. W. \& PALMER, M. E. 1980 The calculation of the thermodynamic properties of propane, propylene, n-butane and ethylene. U.S. Dept Transportation Rep. FRA/ORD76/300. University of Maryland, College Park, MY.

Skripov, V. P. 1974 Metastable Liquids. Wiley.

Strube, H. W. 1971 Numerische untersuchungen zur stabilitat nichtsparisch schwingender blasen. Acustica 25, 289.

TAYLOR, G. I. 1950 The instability of liquid surfaces when accelerated in a direction perpendicular to their planes. I. Proc. R. Soc. Lond. A 201, 192.

Theofanous, T. G. \& Patel, P. D. 1976 Universal relations for bubble growth. Int. J. Heat Mass Transfer 19, 425.

Wakeshima, H. \& Takata, K. 1958 On the limit of superheat. J. Phys. Soc. Japan 13, 1398.

Witte, L. C., Cox, J. E. \& Bouvier, J. E. 1970 The vapor explosion. J. Metals 22, 39. 